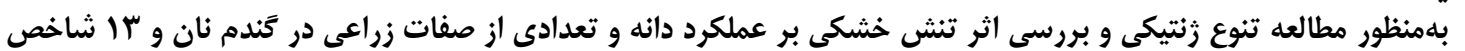

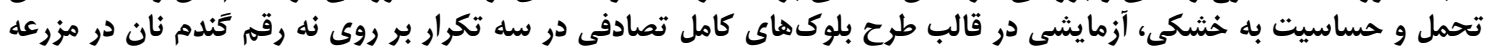

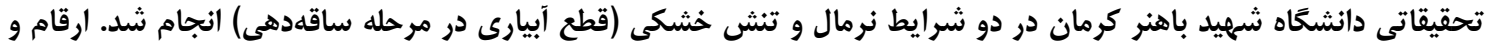

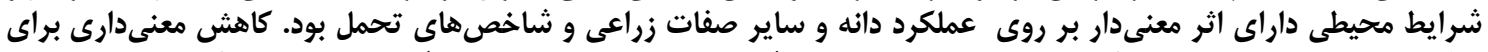

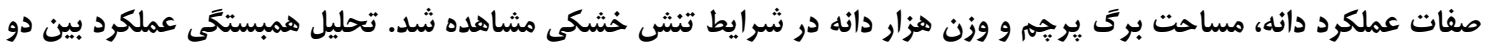

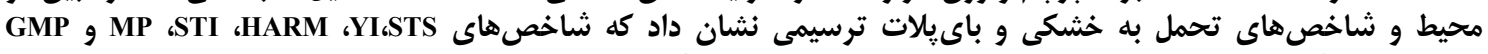

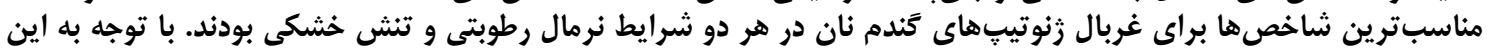

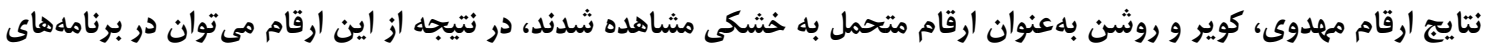
زراعى و اصلاحى استفاده كرد.

وازههاى كليدى: باى يُلات، كَندم نان، تنش خشكى، عملكرد دانه، شاخصهاى تحمل

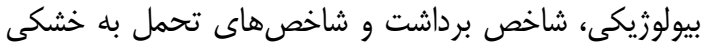

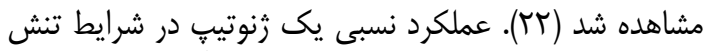

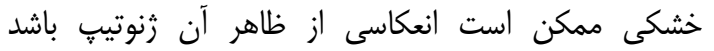

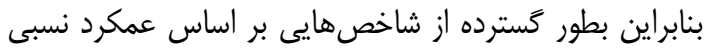

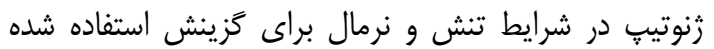

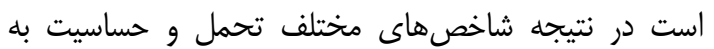

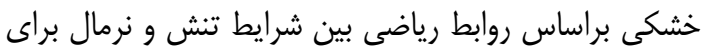

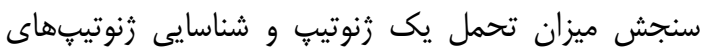

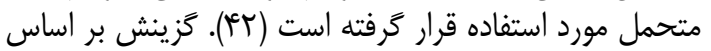

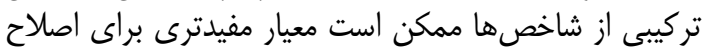

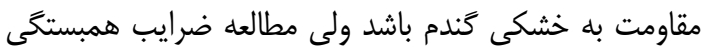

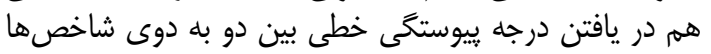

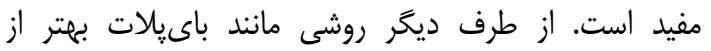

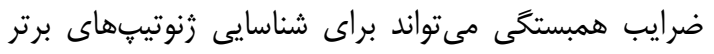

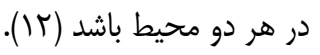

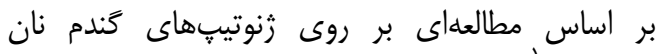

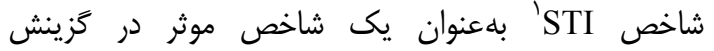

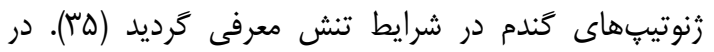

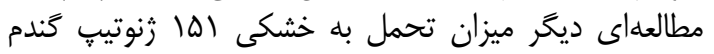

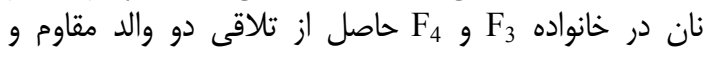
حساس Oste-Gata و

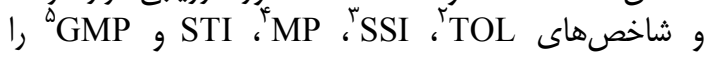

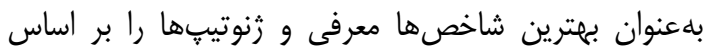

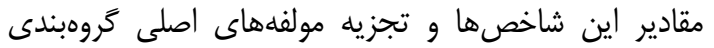

نمودند (1) - (1).

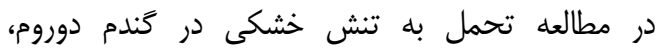

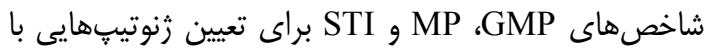
عملكرد بالا در هر دو شرايط تنش و نرمال مناسب تشخيص نوني
مقدمه

جمعيت جهان در حال افزايش است و اهميت توليد مواد

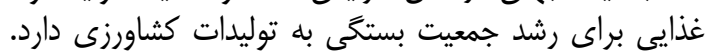

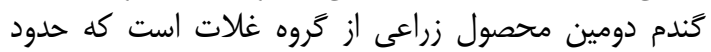

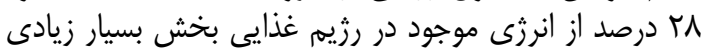

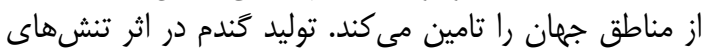

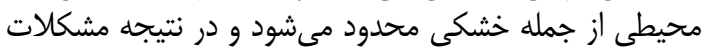

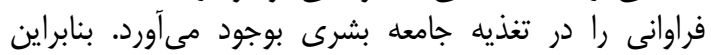

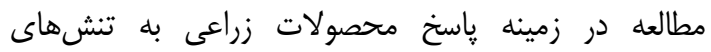
محيطى افزايش يافته است و يكى ازئ از اهداف اصلى اصلى اصلى اصلاح

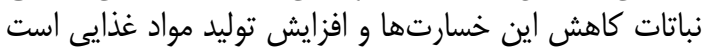

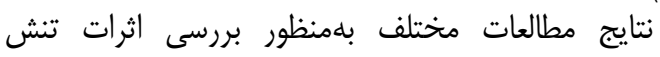

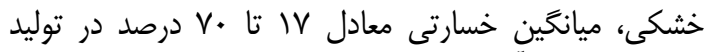

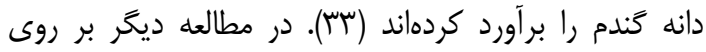

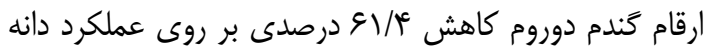

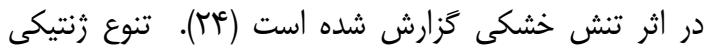
كستردهاى براى صفاتى مثل بيوماس، عملكرد داني شانه، شاخص

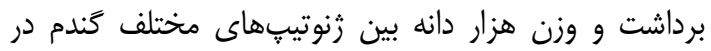

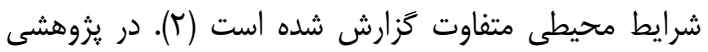

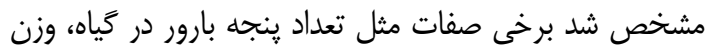

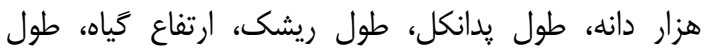

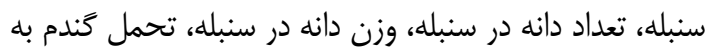
تنش خشكى خاى را تحت تاثير قرار مىدهد

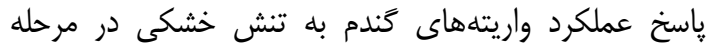

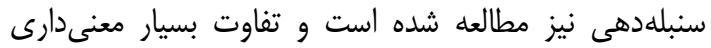

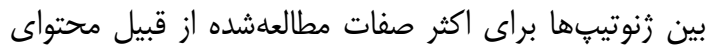

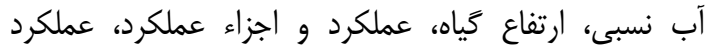




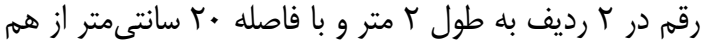

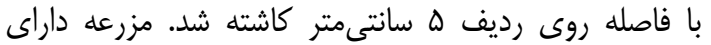

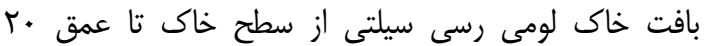

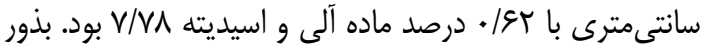

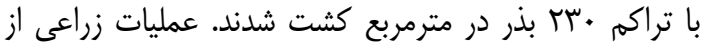

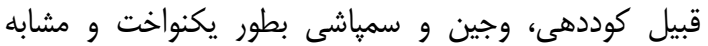

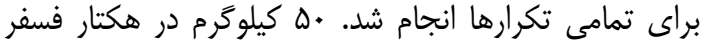

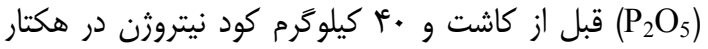

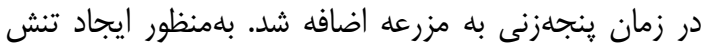
خشكى با توجه به شرايط آب و هوائى منافي منطقه، آبيارى از

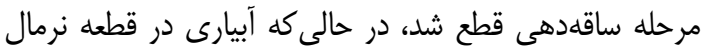

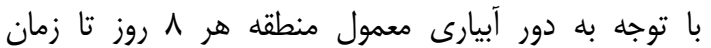

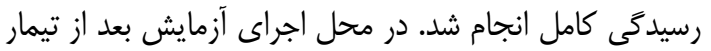

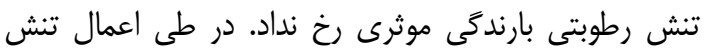

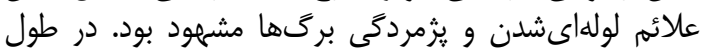
دوره رشد صفات زراعى شامل طول طول ساقد ساقه، تعداد روز

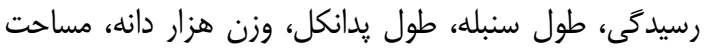

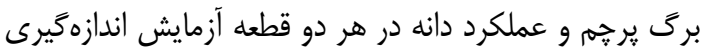

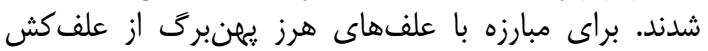

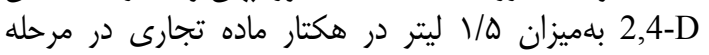

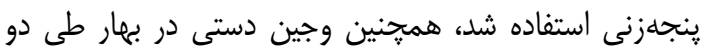

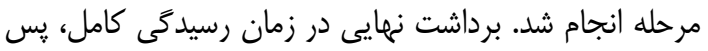

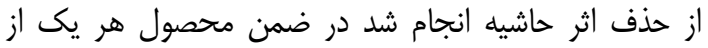

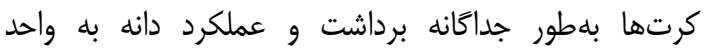

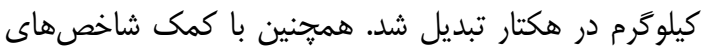

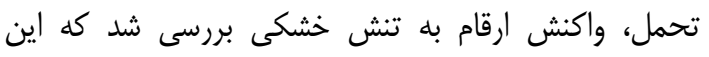

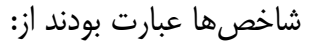

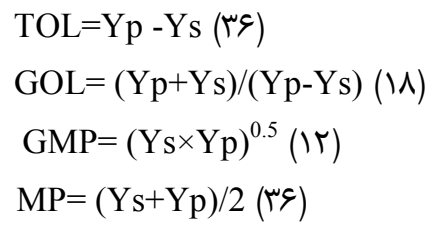

$\mathrm{SSI}=\frac{1-\left(\mathrm{Y}_{\mathrm{S}} / Y_{P}\right)}{S I} \quad S I=1-\left(\frac{Y_{\bar{S}}}{Y_{\bar{P}}}(1)^{\mathrm{F}}\right)$

$\beta(\mathrm{y} / \mathrm{x})=\mathrm{SP}(\mathrm{xy}) / \mathrm{SSx}(\mathrm{l})$

STI $=\frac{\left.\left(\mathrm{Y}_{\mathrm{p}}\right) Y_{S}\right)}{\left(Y_{\bar{P}}\right)^{2}}(\mathrm{Ir})$
$\mathrm{YSI}=\mathrm{YS} / \mathrm{Yp}(\Lambda)$
$\mathrm{YI}=Y S / \overline{\mathrm{Y}} \mathrm{S}(\mathrm{YV})$

$\left.\mathrm{SSPI}=\left({ }^{(p p-Y S} / 2 \overline{Y_{p}}\right) \times 100(\Psi)\right)$

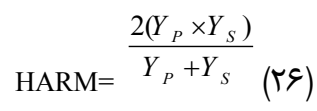

$\mathrm{DRI}=Y S-\widehat{Y S} / \mathrm{SE}(\mathrm{V})$

$S T S=M P+S T I+G M P+Y I+D R I+Y S I-S S I-T O L-\beta()$

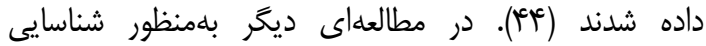

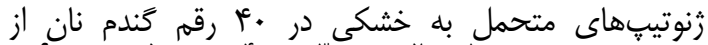

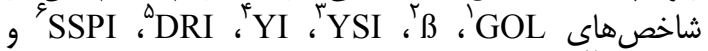
HARM و ورش تجزيه به عاملها استفاده شد و رقهم

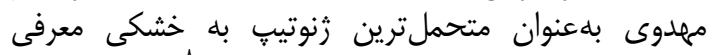

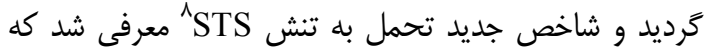

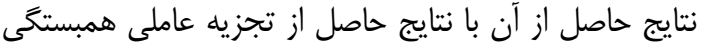

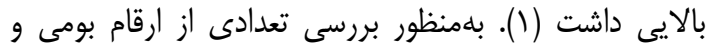

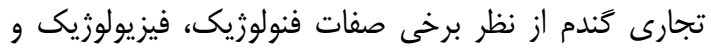

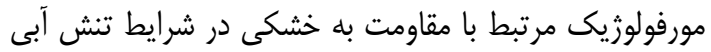

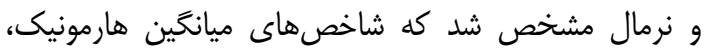

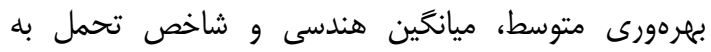

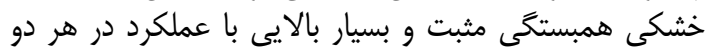

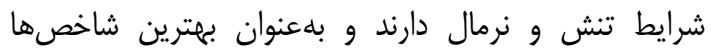

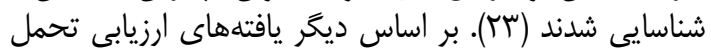

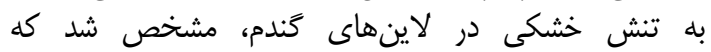

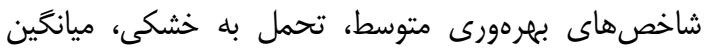

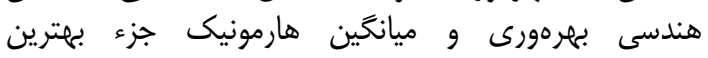
شاخص ها براى غربال كردن در شر ايط تنش و نرمال محسوب

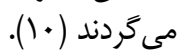

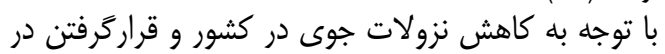

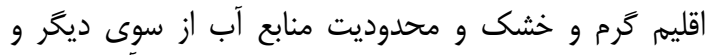

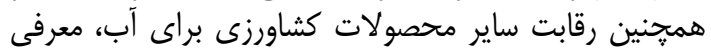

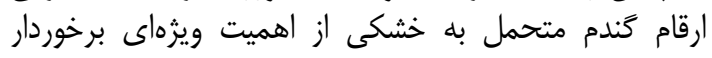

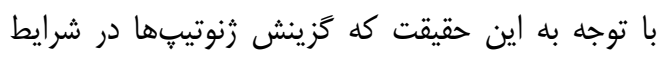

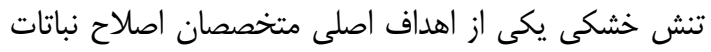

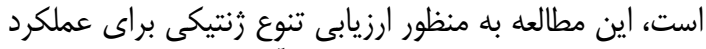

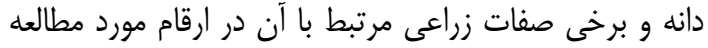

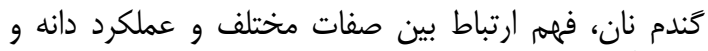

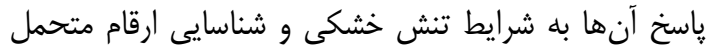

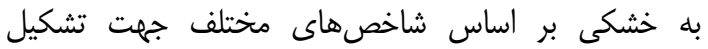

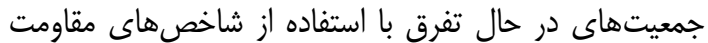
و معرفى بهترين شاخصهال تفا انجام شد.

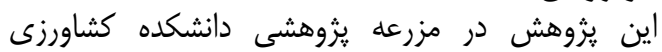

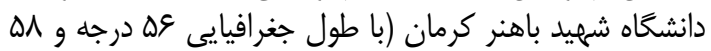

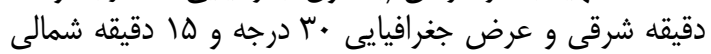

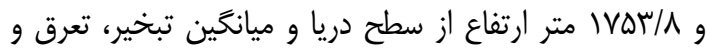

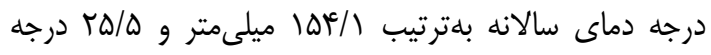

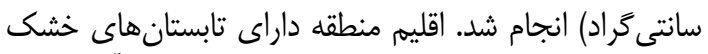

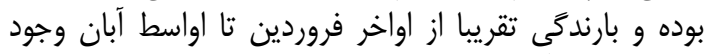

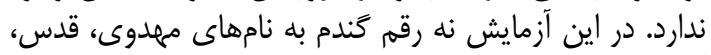

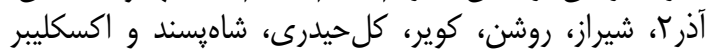

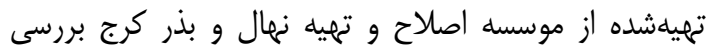

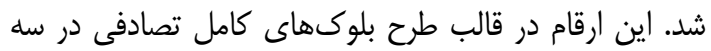

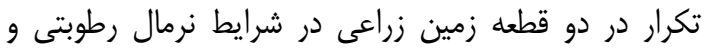

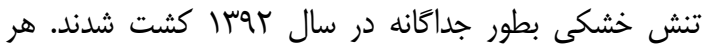

1- Golden Index

5- Drought Resistance Index
2- linear regression coefficient 6- Stress Susceptibility Percentage Index
3- Yield Stability Index

7- Harmonic Index 
نشان دادند تنش خشكى باعث كاهش در اغلب صفات زراعى

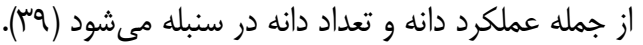

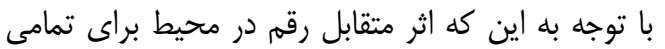

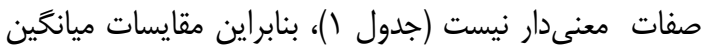

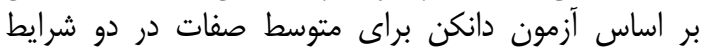

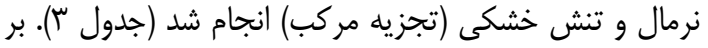

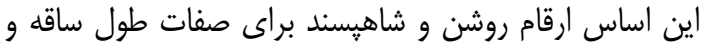

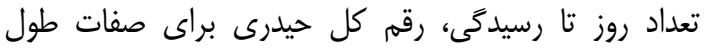

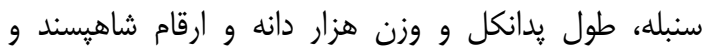

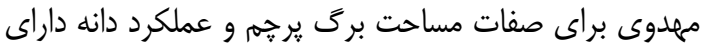

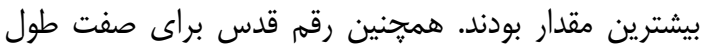

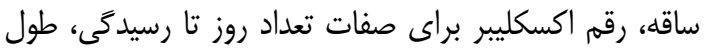

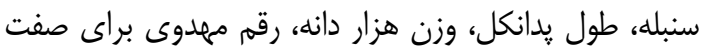

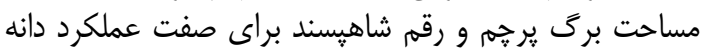

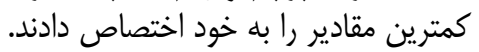

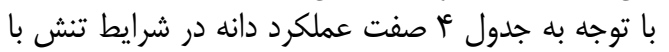

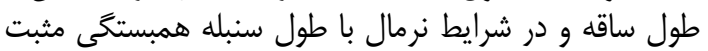

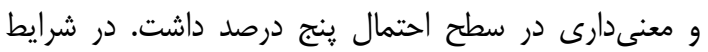

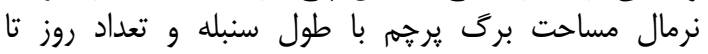

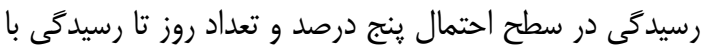

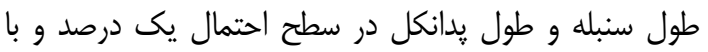

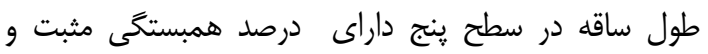

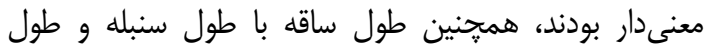

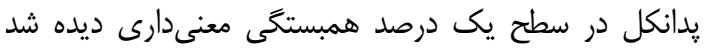

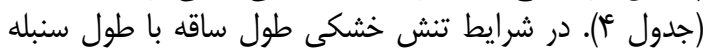

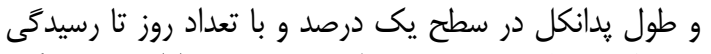

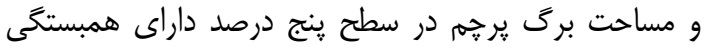

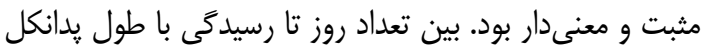

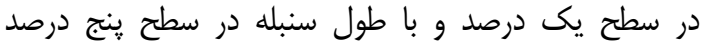

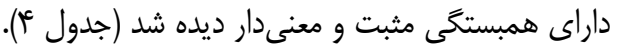

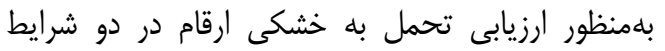

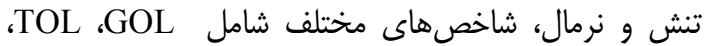

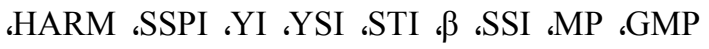

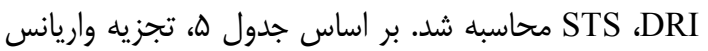

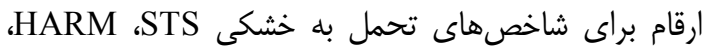
GMP ،SSI ،STI

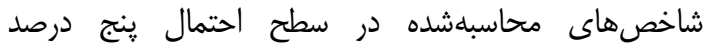

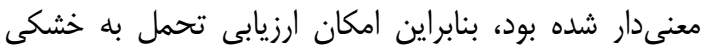

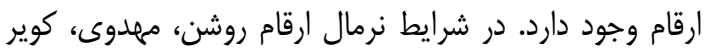

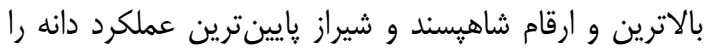

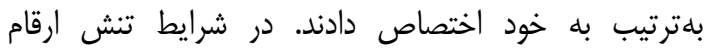

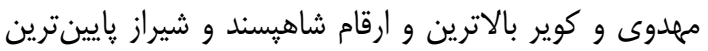

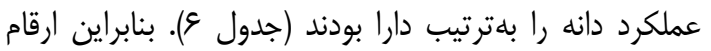

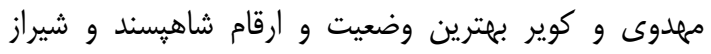

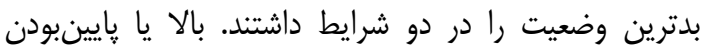

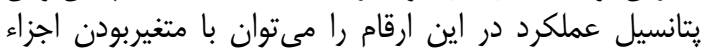

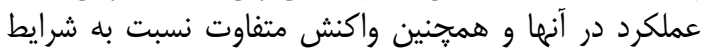
محيطى مرتبط دانست.
براى محاسبه شاخص STS دادههاى خام استفاده نمىشوند بلكه تمام شاخصها در معادله مذكور بايد ابتدا توسط فرمول زير استاندارد شوند:

$$
Z i j=(X i j-\bar{X} i) / S i
$$

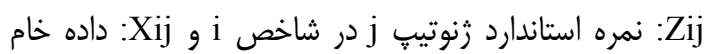

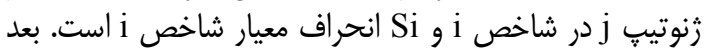

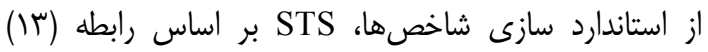

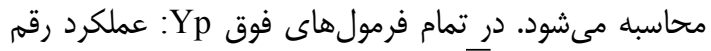
در محيط نرمال، Ys

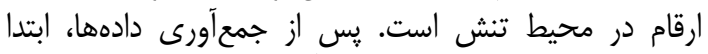

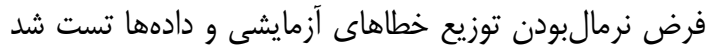

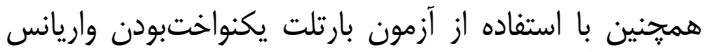
خطاهاى آزمايشى در درون ارقام بررسى شد سيس إنى محاسبه

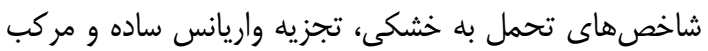

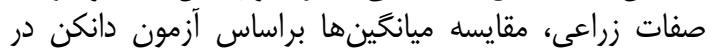

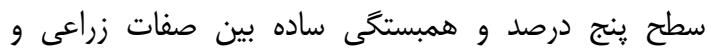

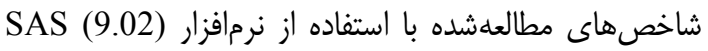

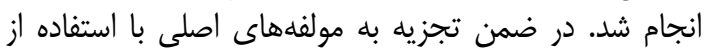

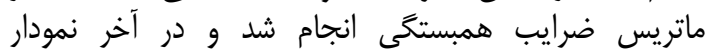

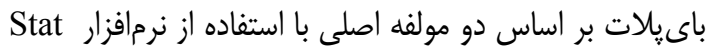
graphics

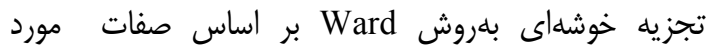
ارزيابى از نرمافزار Minitab استفاده شد.

براساس نتايج تجزيه واريانس مركب (جدول ()، ارقام

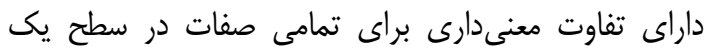

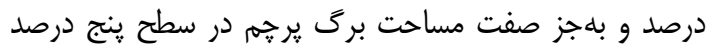

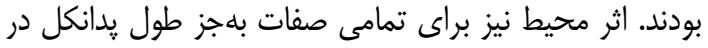

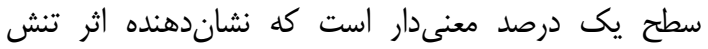

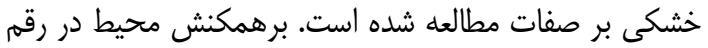

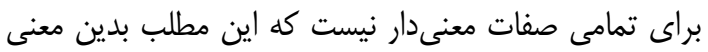

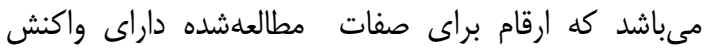
يكسان در محيطهاى متفاوت مىباشند كه با ماندان نتايج ديخر كزارشها مطابقت دارد (س، (1).

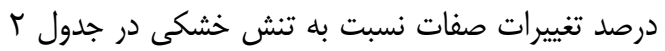

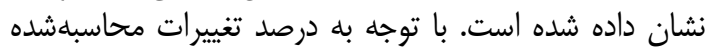

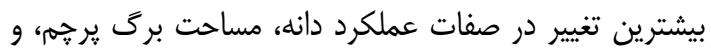

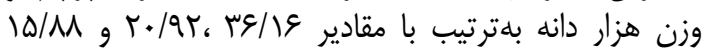

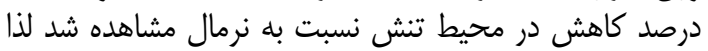

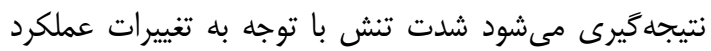

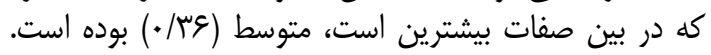

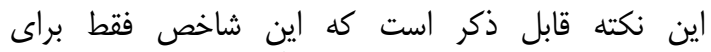
اندازه

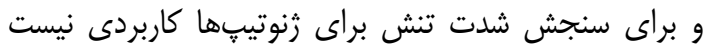

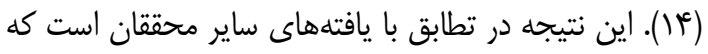


Table 1. Combined ANOVA of traits

\begin{tabular}{|c|c|c|c|c|c|c|c|c|}
\hline 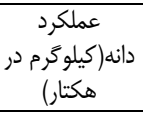 & 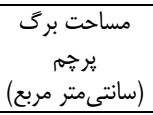 & دانه(گرم) هزار & 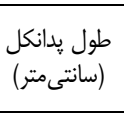 & طول سنتى متر) & رسيدگى(روز) & ساقه(سانتى متر) & درجه آزادى & منبع تغيير \\
\hline$|f| / 9^{\text {***as }}$ & 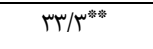 & $|f| / \varphi^{\text {क草 }}$ & $\mathrm{Fr} / \mathrm{V}$ & $1 T / 0^{* \%}$ & $r \cdot / 9^{\text {*ै* }}$ & 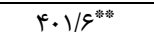 & 1 & محيط \\
\hline 药 & $\mid \omega / \varphi^{*}$ & $r / \Lambda^{*}$ & $I T / V^{\mathrm{ns}}$ & 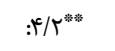 & $r / V^{\mathrm{ns}}$ & $\Delta \Delta / \digamma^{*}$ & f & (محيط) بلوى \\
\hline 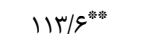 & $\mid \tau / V \Delta^{*}$ & 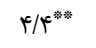 & 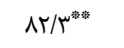 & $9 / 9^{\text {券费 }}$ & $\mid Q / \wedge^{* *}$ & $r \cdot 9 / r^{*}$ & fit & رقم \\
\hline$r T / \Delta$ & $r \cdot / q$ & ואו & 1N/Tq & 1/ & r/AV & १/१ & & ضريب \\
\hline
\end{tabular}

Table 2 . The mean and variation of traits

جدول r- ميانگين و درصد تغييرات صفات

\begin{tabular}{|c|c|c|c|}
\hline درصد تغييرات & ميانكين شرايط تنش خشكى & ميانگين شرايط & صفت \\
\hline$\Delta / v \wedge$ & 19/1 & $9 \% / \Delta$ & طول ساقه (سانتى متر) \\
\hline$F / \Delta)$ & $1 \Lambda Y / 9$ & $191 / 4$ & تعداد روز تا رسيدگى (روز) \\
\hline 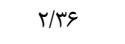 & $1 . / 1$ & $1 \cdot / r$ & طول سنبله (سانتى متر) \\
\hline $\mathrm{V} / \mathrm{V} \wedge$ & rه/A & rN/q & طول پِدانكل (سانتى متر) \\
\hline ( ) & tet/t & GQN/T & عملكرد دانه (كيلوكرم در هكتار) \\
\hline
\end{tabular}

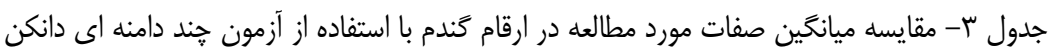
Table 3.Duncan mean comparison of studied traits between wheat cultivars

\begin{tabular}{|c|c|c|c|c|c|c|c|}
\hline (كيلوكَرم در هكتار) & 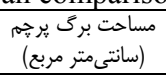 & 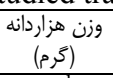 & $\begin{array}{l}\text { طولتيديتر) } \\
\end{array}$ & طولت سنبله & 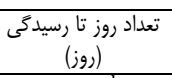 & طولت ساقه & رقم \\
\hline$\Delta 19 / /^{\mathrm{a}}$ & $10 / V^{c}$ & $\Delta \Delta / /^{b c}$ & $r \Delta^{b c}$ & $1 . / /^{\mathrm{ab}}$ & $19 \cdot / 8^{\mathrm{ab}}$ & $\Lambda V / \kappa^{c}$ & مهدوى \\
\hline$r \notin \varepsilon / T^{a b c}$ & $\mathrm{IV} / \mathrm{v}^{\mathrm{bc}}$ & $\varphi \& / \Lambda^{\mathrm{cd}}$ & $r \mu / g^{a b}$ & $N / \kappa^{c d}$ & $191 / \mu^{a b}$ & $q \uparrow / \mathrm{rabc}^{\mathrm{abc}}$ & آذر r \\
\hline$\Delta \cdot r / \Delta^{a b}$ & $r \tau / c^{b c}$ & $\Delta F / F^{b c}$ & ()$^{a b}$ & $9 / q^{b c}$ & $19 \cdot / \mu^{a b}$ & $111 / 1^{a}$ & روشن \\
\hline$r q q / r^{b c}$ & $r c^{\mathrm{ab}}$ & $9 \cdot 1^{\mathrm{ab}}$ & $\widetilde{r} / q^{\mathrm{ab}}$ & $11^{\mathrm{ab}}$ & $19 T / \mathrm{ra}^{\mathrm{a}}$ & $\Lambda \mathrm{V} / \mathrm{N}^{\mathrm{c}}$ & قدس \\
\hline$q \cdot V / \mathrm{r}^{\mathrm{a}}$ & $r \mu^{\mathrm{ab}}$ & $91 / 1^{a b}$ & $\widetilde{r} / \Delta^{\mathrm{ab}}$ & $11^{\mathrm{ab}}$ & $19 r^{\mathrm{a}}$ & $a \cdot / v^{b c}$ & كوير \\
\hline$\uparrow \Psi q / \Delta^{a b c}$ & $r / s^{b c}$ & $\mathrm{Fr} / \mathrm{A}^{\mathrm{d}}$ & $T N / \Lambda^{c}$ & $V / x^{d}$ & $M F / \mu^{b}$ & $s g / V^{\mathrm{d}}$ & اكسكليبر \\
\hline $\mathrm{rva} / \mathrm{v}^{\mathrm{abc}}$ & $\mid V / g^{b c}$ & $\varepsilon \varphi / V^{\mathrm{a}}$ & $r r^{a}$ & $1 r^{\mathrm{a}}$ & $19 \cdot / 8^{\mathrm{ab}}$ & $1.9^{\mathrm{abc}}$ & كل حيدى \\
\hline rafbc & $r \cdot /)^{b c}$ & $\Delta q / v^{a b}$ & $r q / \mu^{a b}$ & $1 /^{a b}$ & $19 x^{\mathrm{a}}$ & $q / / \mathrm{wabc}^{2}$ & شيراز \\
\hline$r \Delta F / T^{c}$ & $r \cdot / r^{a}$ & $g r / \Lambda^{\mathrm{ab}}$ & $\kappa r / \Lambda^{a b}$ & $W / \Delta^{a b}$ & $198^{\mathrm{a}}$ & $1.9 / 1^{a b}$ & شاهِيند \\
\hline
\end{tabular}

Table 4.The correlation coefficients of traits between wheat cultivars

$$
\text { جدول ع- ضرايب همبستخى صفات در ارقام گندم نان }
$$

\begin{tabular}{|c|c|c|c|c|c|c|c|c|c|}
\hline \multirow{8}{*}{$\begin{array}{l}\frac{3}{3} \\
3 \\
3 \\
3 \\
3 \\
3 \\
3\end{array}$} & $\mathrm{v}$ & 9 & $\Delta$ & f & $r$ & $r$ & 1 & & صفت \\
\hline & $\cdot /\left.4\right|^{\prime \prime}$ &.$/\left.4\right|^{*}$ & $\cdot / / r^{n s}$ &.$/ 9 \Lambda^{* * 3}$ &.$/ 9^{* * *}$ & $\cdot / \Gamma \omega^{*}$ & 1 & & 1 \\
\hline & $\cdot|r|^{\mathrm{ns}}$ & $\cdot / r \Delta^{\mathrm{ns}}$ &.$/ r^{n s}$ & $\cdot|\Delta|^{* \text { *⿻ }}$ & $.149^{*}$ & 1 & . & $q$ & $r$ \\
\hline &.$/ \mathrm{rns}^{\mathrm{ns}}$ & . & $\cdot / \alpha^{\mathrm{ns}}$ & . $/ \pi \varepsilon^{\mathrm{ns}}$ & 1 & 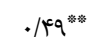 & $\cdot 10^{* * *}$ & s & $r$ \\
\hline & $\cdot / \cdot r^{\mathrm{ns}}$ & $\cdot / N e^{\mathrm{ns}}$ & $-\cdot / \cdot e^{\mathrm{ns}}$ & 1 & 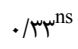 & $\cdot / \Delta r^{* * * * *}$ & . $/ \Lambda r^{* *: *}$ & है. & r \\
\hline & $\cdot / 18^{\mathrm{ns}}$ & $-\cdot / 1 Q^{\mathrm{ns}}$ & 1 & $\cdot / / v^{\mathrm{ns}}$ & $\cdot / \cdot 1^{\mathrm{ns}}$ & $-\cdot / / r^{\mathrm{ns}}$ & $\cdot \pi e^{\mathrm{ns}}$ & $\frac{E}{\xi}$ & 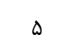 \\
\hline & $\cdot \mid r r^{n s}$ & 1 & $-\cdot 1 \cdot \Lambda^{\mathrm{ns}}$ & $\cdot 1 \cdot \Delta^{\mathrm{ns}}$ & $\cdot / \mathcal{F} V^{*}$ & $\cdot / \mathbb{r}^{* *}$ &.$/ 18^{\mathrm{ns}}$ & & \& \\
\hline & 1 & . & $\cdot / . r^{\mathrm{ns}}$ & $-\cdot 1 \cdot 0^{\mathrm{ns}}$ & $.144^{\prime \prime}$ &.$/ 18^{\mathrm{ns}}$ &.$/ r^{\mathrm{ns}}$ & & v \\
\hline
\end{tabular}


Table 5. The ANOVA of drought tolerance indices between wheat cultivars

\begin{tabular}{|c|c|c|c|c|c|c|c|c|}
\hline \multicolumn{3}{|c|}{ خطا } & \multicolumn{2}{|c|}{ رقم } & \multicolumn{2}{|c|}{ بلوك } & \multicolumn{2}{|c|}{ منبع تغيير } \\
\hline \multicolumn{3}{|c|}{19.48} & \multicolumn{2}{|c|}{ rq9s } & \multicolumn{2}{|c|}{$\Lambda r \cdot \Lambda \Lambda^{* * *^{*}}$} & \multicolumn{2}{|c|}{ TOL } \\
\hline \multicolumn{3}{|c|}{ ME. } & \multicolumn{2}{|c|}{ gr. * } & \multicolumn{2}{|c|}{$\left.1 \ldots\right|^{* w^{*}}$} & \multicolumn{2}{|c|}{ GOL } \\
\hline \multicolumn{3}{|c|}{1.141} & \multicolumn{2}{|c|}{ FTERT } & \multicolumn{2}{|c|}{ 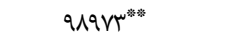 } & \multicolumn{2}{|c|}{ GMP } \\
\hline \multicolumn{3}{|c|}{ IIIfT } & \multicolumn{2}{|c|}{ Truv" } & \multicolumn{2}{|c|}{ 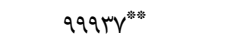 } & \multicolumn{2}{|c|}{ MP } \\
\hline \multicolumn{3}{|c|}{$\cdot / 1 Q$} & \multicolumn{2}{|c|}{$\cdot / \Gamma \Delta^{* *^{*}}$} & \multicolumn{2}{|c|}{$\cdot / \wedge \Upsilon^{\mu^{*} \cdot w^{*}}$} & \multicolumn{2}{|c|}{ SSI } \\
\hline \multicolumn{3}{|c|}{$\cdot / \Lambda$} & \multicolumn{2}{|c|}{$r / \Lambda^{* *}$} & \multicolumn{2}{|c|}{$r / q^{* * *}$} & & \\
\hline & $\cdot 1 \cdot 1$ & & & & & & & \\
\hline & щᄉ. & & & & & & & \\
\hline & १९१ & & & & & & & \\
\hline & אזו & & & & & & & \\
\hline & DNTITI & & & & & & & \\
\hline & $\cdot 18 \mathrm{~V}$ & & & & & & & \\
\hline & $r g / V$ & & & & & & & \\
\hline & $M$ & & & & & & & \\
\hline Table 6. & ight tole & indices & en wh & tivars & مد نان & كى درصد & ر تحمل & 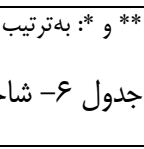 \\
\hline $\mathrm{Yp}$ & Ys & TOL & GOL & GMP & MP & SSI & $\beta$ & رقم \\
\hline$V V V^{c} / r$ & $019 / 1$ & $r \Delta \omega / 1$ & $\Delta / .9$ & grr/q & ste/V & $\cdot / 9$ & .109 & مهدوى \\
\hline$g r \cdot / r$ & Feg/T & $198 / 1$ & $\Delta / \Delta Q$ & $\Delta K F / \Delta$ & WTH & ./AT &.$/ 94$ & آذر r \\
\hline$\Lambda \cdot r / \Delta$ & $\Delta \cdot r / \Delta$ & $r \cdot 1$ & rr/r & $g r \Delta / \kappa^{c}$ & gQT & $1 / . r$ & س & روشن \\
\hline$V Q \cdot / \Lambda$ & $G \cdot V / r$ & $\mid f r / F$ & $9 / 48$ & SVQ/T & $9 \vee 9 / 1$ & $\cdot / \Delta T$ & $1 / \cdot F$ & كوير \\
\hline qr. & $r q q / r$ & & $r / r q$ & $F \| / \Lambda$ & $F+q / g$ & $1 / \Delta S$ & 1/49 & قدس \\
\hline س/m & $r+q / D$ & $I V / V$ & $81 \cdot 0$ & $019 / 1$ & $\Delta T E / T^{C}$ & $\cdot / \mathrm{Vr}$ & $\cdot|r|$ & اكسكليبر \\
\hline SIr/A & frq/V & Irf & $N / 10$ & DFT/G & DFE/V & .109 & $1 / 1 V$ & كلحيدرى \\
\hline$\Delta G T / \Gamma$ & ref & $r q / \mu$ & $r / V V$ & rND/r & $F \mid r / l$ & $1 /$ Fe $^{4}$ & $1 / 14$ & شيراز \\
\hline 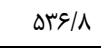 & $r \Delta F / T$ & TAT/D & $r / v q$ & rsq/r & $\Gamma 9 \Delta / \Delta$ & מאוא & $1 / 7 q$ & شاهيسند \\
\hline STI & YSI & YI & SSPI & HARM & DRI & STS & & رقم \\
\hline $1 / .9$ & $.19 \mathrm{~V}$ & $1 / \mu$ & $\cdot / T$ & GTI/D & $1 / 10$ & $v / v q$ & & مهدوى \\
\hline$\cdot / V^{2}$ & $\cdot / V$ & $1 / 1 r$ & $\cdot / 10$ & $\Delta T \Delta / q$ & $\cdot / 19 q$ & $r / v$ & & آذر r \\
\hline 1 & .180 & $1 / T V$ & $\cdot / T^{E}$ & $8 / N / r$ &.$/ I V$ & $g / r V$ & & روشن \\
\hline $1 / I V$ & . $/$ Ar & $1 / \Delta r$ & .111 & $s V I / F$ & •/1AD & $V / \Delta S$ & & كوير \\
\hline سז/. & $\cdot / \Delta \Lambda$ & $\cdot \mid 8 \Lambda$ & $\cdot / 4 \Lambda$ & $r V / r$ & -.1 .9 & $-q / \cdot r$ & & قدس \\
\hline .199 & $\cdot / v^{c}$ & $1 / 11$ & سו/. & DIT & $\cdot / V \Delta$ & $\Delta / 11$ & & |كسكليبر \\
\hline$\cdot / V \Delta$ & $\cdot|\wedge|$ & $1 / \pi 1$ & $\cdot / 1$ & $\Delta H N / \Delta$ & / & $1 / .9$ & & كل حيدرى \\
\hline . & $\cdot / 4 V$ & .199 & שr/. & rఎq/r & -.1 .9 & $-q / \cdot v$ & & شيراز \\
\hline . & $\cdot / \& \wedge$ & - /at & . & med & $-\cdot / \mu F$ & $-1 \cdot / \Delta T$ & & شاهيسند \\
\hline
\end{tabular}

شيراز حساس و بر اساس شاخصهاى DRI و

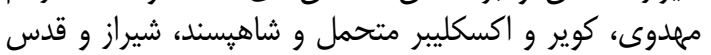

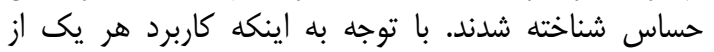

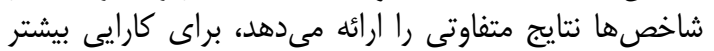

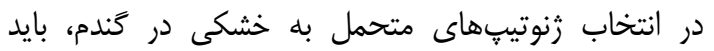

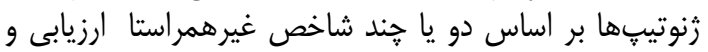

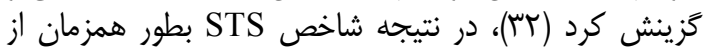

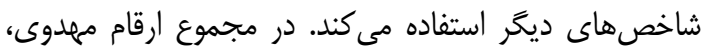

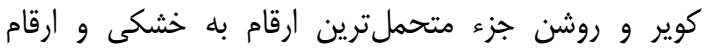

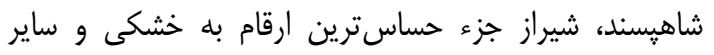
ارقام در سطح متوسط تشخيص دادئ شداء شدند.
با توجه به نتايج جدول 9 بر اساس شاخصهاى TOL

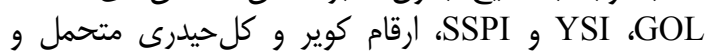

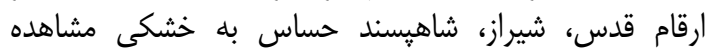

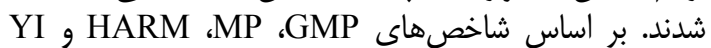

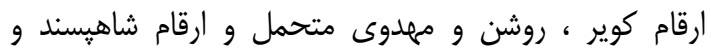

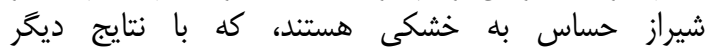

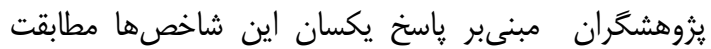

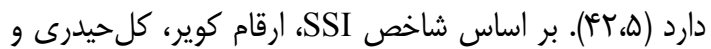

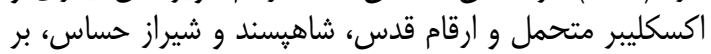

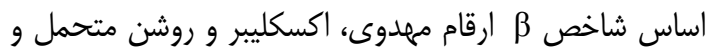

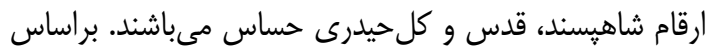
شاخص STI ارقام كوير و مهدوى ميدى متحمل و شاهيسند و و 
حساس جدا كند. زنوتيڤ هاى انتخابشده بر اساس اين

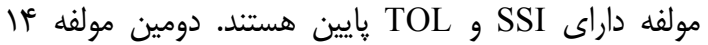

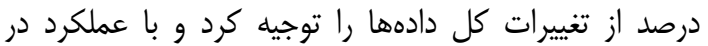

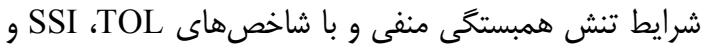

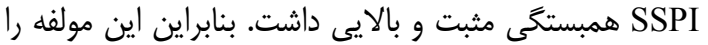

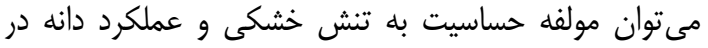

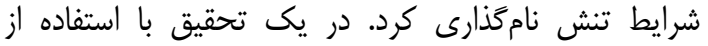

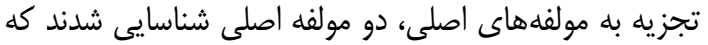

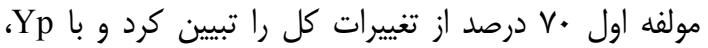

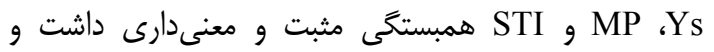

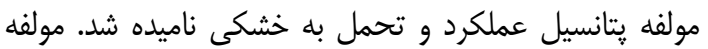

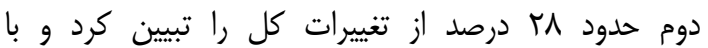

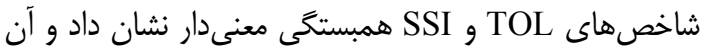

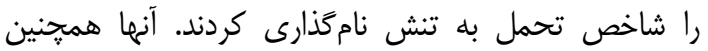

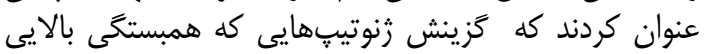

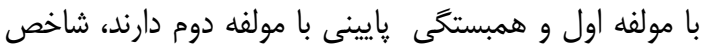

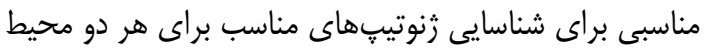

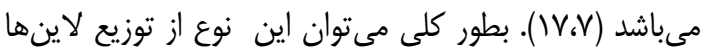

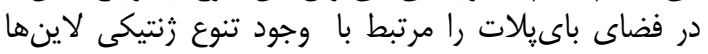

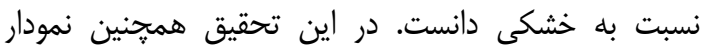

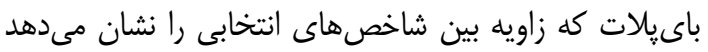

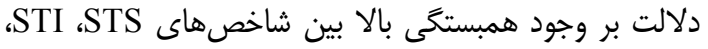

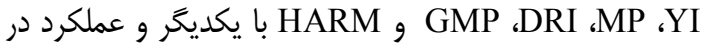

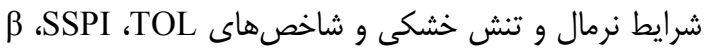

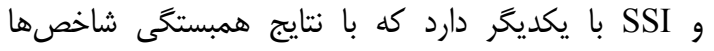

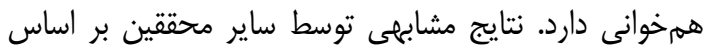

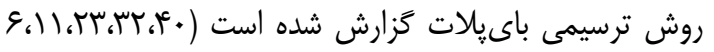

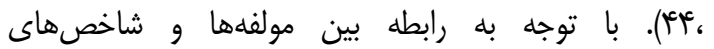

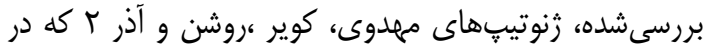

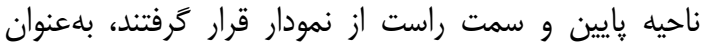

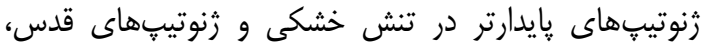

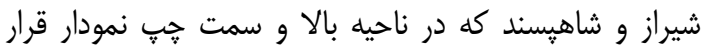

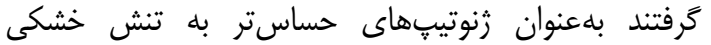

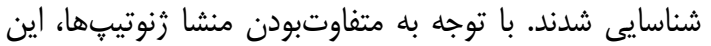

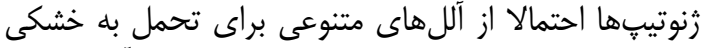

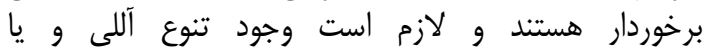

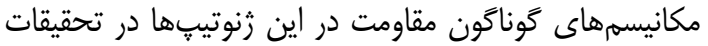
آينده بررسى شود.

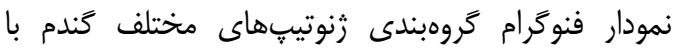

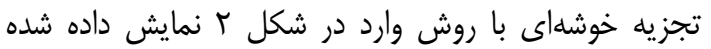

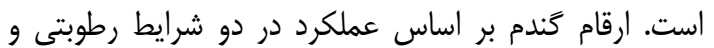

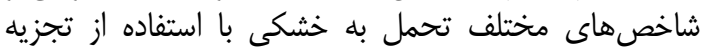

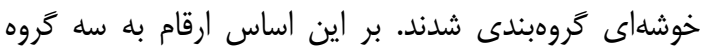

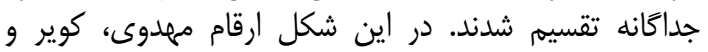

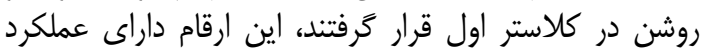

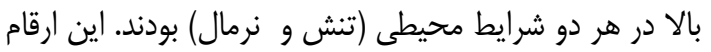

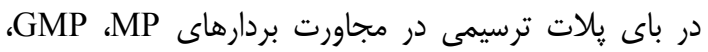

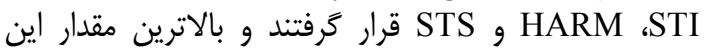
شاخصها را به خود اختصاص دادند. در نتيجه اين ارقام
با توجه به جدول Vا، همبستكى بالا و معنىدارى بين

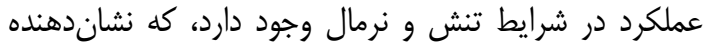

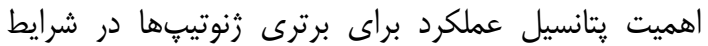

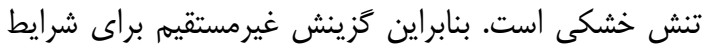

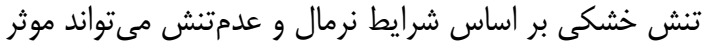

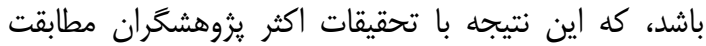

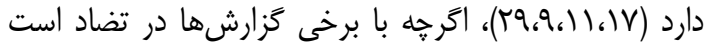

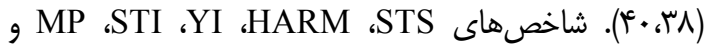
داراى همبستخى مثبت و معنى دارى با عملكرد در هر كر

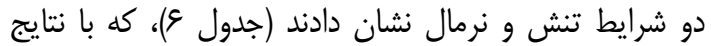

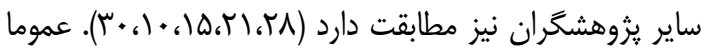

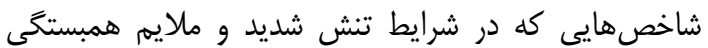

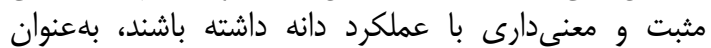

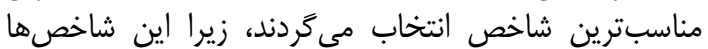

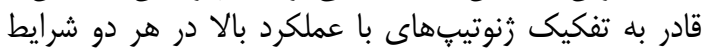

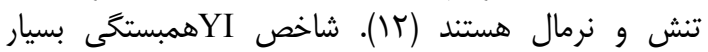

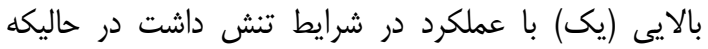

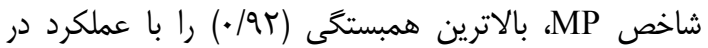

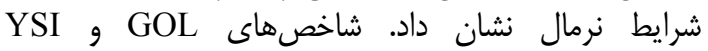

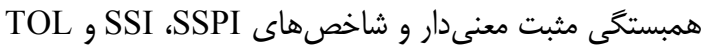

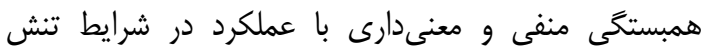

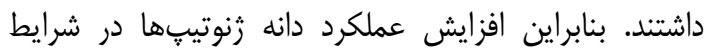

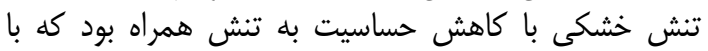

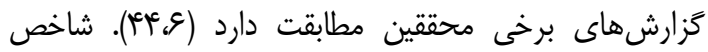

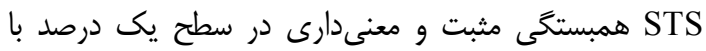

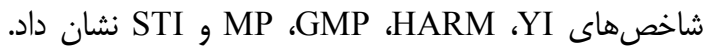

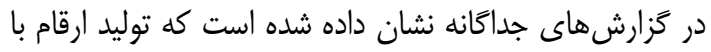

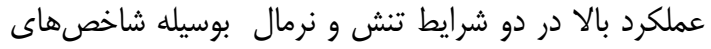

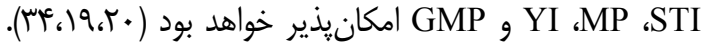

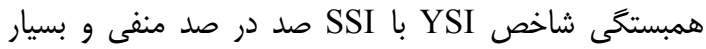

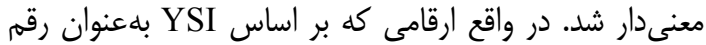

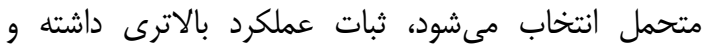

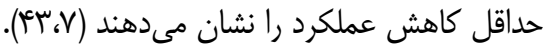

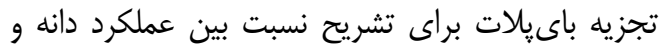

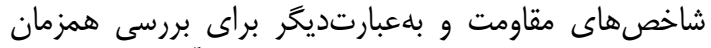

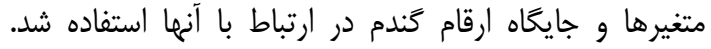

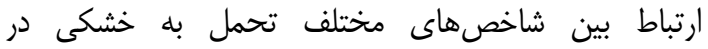

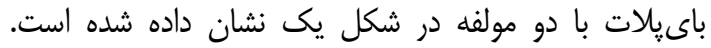

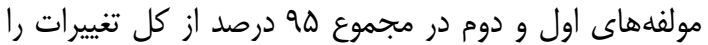

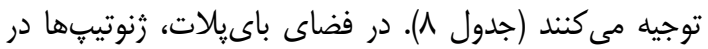

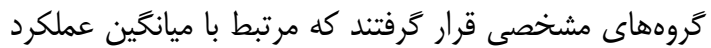

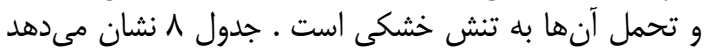

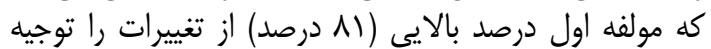

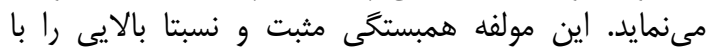

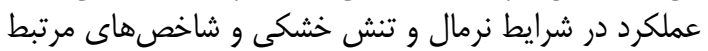

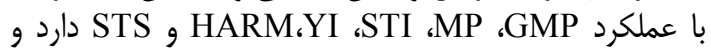

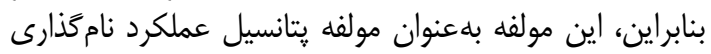

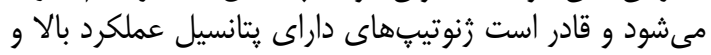

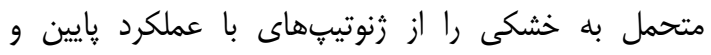


دانه و ساير صفات زراعى را تحت تاثير قرار داده است. كاهش

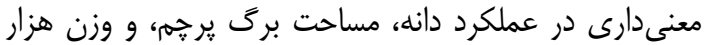

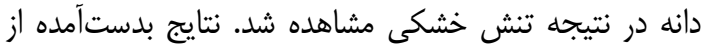

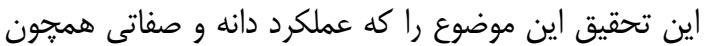

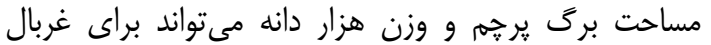

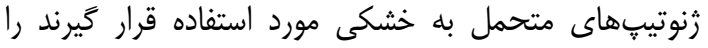

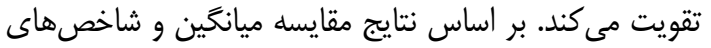

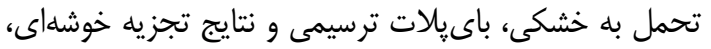

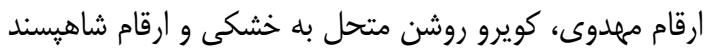

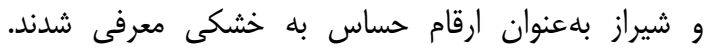

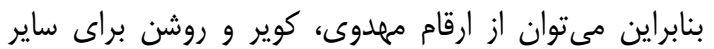

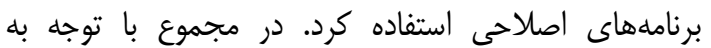

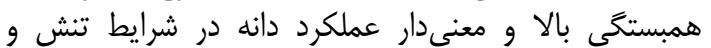

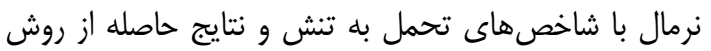

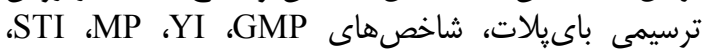

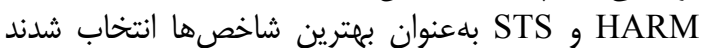

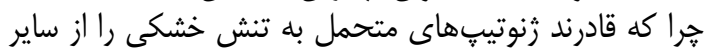

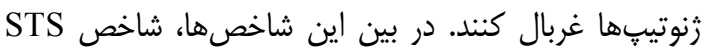
مناسبترين شاخص براى اين منظور شناخته شد.
بلهنوان زنوتيڤهاى متحل به تنش با عملكرد پايدار و بالا

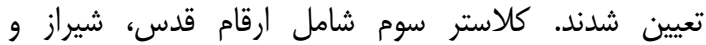

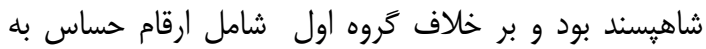

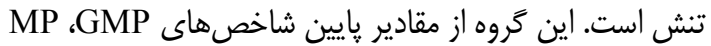

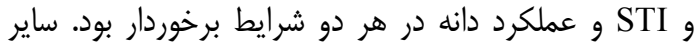

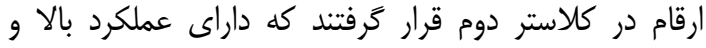

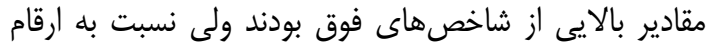

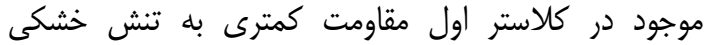

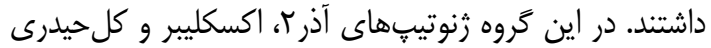

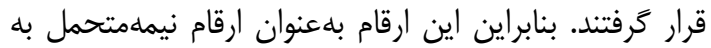

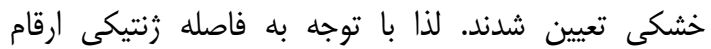

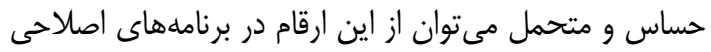

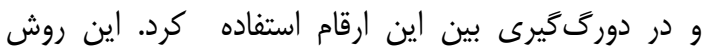

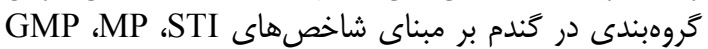
و توسط ساير يُوهشكران نيز استفاده شده است

در اين مطالعه تنوع زنتيكى براى عملكرد دانه و ساير

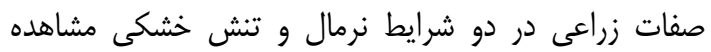
شد. نتايج نشان داد كه محيط بلهصورت معنى دارى عملكرد

Table 7. The correlation coefficients of drought tolerance indices between wheat cultivars

\begin{tabular}{|c|c|c|c|c|c|c|c|c|c|c|c|c|c|c|}
\hline & STS & DRI & HARM & SSPI & YI & YSI & STI & $\beta$ & SSI & MP & GMP & GOL & TOL & Ys \\
\hline Yp & $\cdot / V^{\prime \prime \prime}$ & $\cdot / K^{n s}$ & $\cdot / \Lambda \mathcal{E}^{* * *}$ & $-\cdot 1 \cdot 9^{\mathrm{ns}}$ & $\cdot / V \Lambda^{* * a}$ & $\cdot / 4 \Delta^{\mathrm{ns}}$ & $.19^{* *}$ & $-\cdot / 4 \Lambda^{\mathrm{ns}}$ & $\cdot / A^{\mathrm{n}} \omega^{\mathrm{ns}}$ & $\cdot / 9 r^{* * *}$ & $\cdot / 19^{* * *}$ & $\cdot / \xi^{n s}$ & $-\cdot 1 \cdot 8^{\mathrm{ns}}$ & $\cdot / V \Lambda^{*}$ \\
\hline Ys & $\cdot / 98^{* \# *}$ & $\left.\cdot()^{\mathrm{n}}\right)^{\mathrm{ns}}$ & $\cdot / 9 \Lambda^{* * *}$ & $-\cdot / \& V^{*}$ & $1^{* * *}$ & $\cdot / 9^{* * *}$ & $\cdot / 98^{* *: *}$ & $-\cdot / \mu^{\mathrm{ns}}$ & $-\cdot / 9^{* * n}$ & $\cdot / 98^{* * * *}$ & $\cdot / 9 V^{* h a}$ & 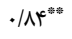 & $-\cdot / 8 \mathrm{~V}^{*}$ & 1 \\
\hline TOL & $-\cdot / / \overline{v^{n s}}$ & $-\cdot / 1 \varepsilon^{\mathrm{ns}}$ & $-\cdot / \Delta \Delta^{\mathrm{ns}}$ & 1 & $-.19 V^{*}$ & $-. / 9)^{* * *}$ & $-\cdot / \mathrm{eq}^{\mathrm{ns}}$ & $-\cdot / \cdot \wedge^{\mathrm{ns}}$ & $\cdot(9)^{* * * *}$ & $-\cdot / \psi^{n}$ ns & $-\cdot / 0^{\mathrm{ns}}$ & $. / 9)^{*}$ & 1 & \\
\hline GOL & $-\cdot / V \Lambda^{*}$ & $\cdot / M r^{\mathrm{ns}}$ & $\cdot / V \Delta^{*}$ & $-\cdot / 9)^{\frac{* a}{*}}$ & 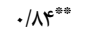 & $\cdot / 90^{* h}$ & $\cdot / V^{*}$ & $\cdot / \cdot \wedge^{\mathrm{ns}}$ & $\cdot / 90^{\text {**a }}$ &.$/ 9 V^{*}$ & $\cdot / V r^{*}$ & 1 & & \\
\hline GMP & $\cdot / 9)^{* * *}$ & $\cdot / \phi^{\mathrm{ns}}$ &.$/ 99^{* * *}$ & $-\cdot / \Delta^{\mathrm{ns}}$ & $\cdot / 9 V^{* a n}$ & $\cdot / \Lambda^{* * *}$ & $\cdot / 99^{* * *}$ & $-\cdot / \mu^{n s}$ & $-\cdot / \Lambda^{* \frac{m^{2}}{2}}$ &.$/ 99^{* * *}$ & 1 & & & \\
\hline MP & $\cdot(9)^{* * a}$ & $\cdot / \mathrm{er}^{\mathrm{ns}}$ & $\cdot / 99^{* * *}$ & $-\cdot / \psi^{n n s}$ & $\cdot / 99^{* * *}$ & $\cdot / V \Delta^{*}$ & $\cdot / 99^{* * * \omega^{*}}$ & $-\cdot / /^{\mathrm{ns}}$ & $-\cdot / V \Delta^{*}$ & 1 & & & & \\
\hline SSI & $-\cdot / 9^{* * *}$ & $-\cdot / \Gamma \omega^{\mathrm{ns}}$ & $-\cdot / \Lambda \Lambda^{* * *}$ & $. / 9)^{* a n}$ & $-\cdot / 9^{* * *}$ & $-1^{* n}$ & $-\cdot / V V^{*}$ & $\cdot / / v^{\mathrm{ns}}$ & 1 & & & & & \\
\hline$\beta$ & $-\cdot /<q^{n s}$ &.$- / 9 \Psi^{m *}$ & $-\cdot / \Gamma \Lambda^{\mathrm{ns}}$ & $-\cdot 1 \cdot 1^{\mathrm{ns}}$ & $-\cdot / \mathrm{r}^{\mathrm{ns}}$ & $-\cdot / / v^{n s}$ & $-\cdot / \Gamma \omega^{n s}$ & 1 & & & & & & \\
\hline STI & $\cdot(9)^{\frac{* a}{*}}$ & $\cdot /\left.\right|^{\mathrm{nss}}$ &.$/ 99^{* * *}$ & $-\cdot / 4 v^{n s}$ & $\cdot / 98^{* a t}$ & $\cdot / V V^{*}$ & 1 & & & & & & & \\
\hline YSI &.$/ 9^{*}$ & $\cdot / \Gamma \Delta^{\mathrm{ns}}$ & $\cdot / \Lambda \mu^{*}$ & $-\cdot / 9)^{\frac{* 0}{*}}$ & $\cdot 19^{* * *}$ & 1 & & & & & & & & \\
\hline YI & $\cdot / 98^{* * *}$ & $\left.\cdot /{ }^{4}\right)^{\mathrm{ns}}$ & $\cdot / 9 \Lambda^{* * *}$ & $-\cdot / \& V^{*}$ & 1 & & & & & & & & & \\
\hline SSPI & $-\cdot / \& \mathrm{~V}^{*}$ & $-\cdot / 18^{\mathrm{ns}}$ & $-\cdot / \Delta \Delta^{\mathrm{ns}}$ & 1 & & & & & & & & & & \\
\hline HARM & $\cdot / 90^{* \#}$ & $\cdot /{ }^{e} \Delta^{\mathrm{ns}}$ & 1 & & & & & & & & & & & \\
\hline DRI & $\cdot \mid(4)^{\mathrm{ns}}$ & 1 & & & & & & & & & & & & \\
\hline STS & 1 & & & & & & & & & & & & & \\
\hline
\end{tabular}


Table 8. The eigen value of drought tolerance indices in principle component analysis

جدول ^-مقادير ويزه شاخصهاى تحمل در تجزيه به مولفهاى اصلى

\begin{tabular}{|c|c|c|}
\hline PCA2 & PCA1 & مولفه \\
\hline T/Rg & $11 / \% q$ & مقدار ويثه \\
\hline.$/ 90$ & $\cdot|\wedge|$ & سهم تجمعى \\
\hline.$/ T r$ &.$/ 41$ & YP \\
\hline.$- / M F$ &.$/ \% 9$ & YS \\
\hline re & $-\cdot / r \mid$ & TOL \\
\hline.$- / \mu$ & . $/{ }^{\prime} \Delta$ & GOL \\
\hline.$/ I V$ &.$/ \% \Lambda$ & GMP \\
\hline$-\cdot / \cdot \theta$ & و & MP \\
\hline . Kt & $-\cdot / T V$ & SSI \\
\hline سז/.- &.$- / 11$ & $\beta$ \\
\hline.$/ 19$ & $\cdot / \uparrow \wedge$ & STI \\
\hline$-. \cdot / r$ & ع & YSI \\
\hline $.1 \cdot 4$ & ./\%q & YI \\
\hline$\cdot / F \Delta$ & $-\cdot|r|$ & SSPI \\
\hline.$/ 1 f$ & $\cdot / \uparrow \wedge$ & HARM \\
\hline.$/ 19$ & r/א. & DRI \\
\hline $.1 \cdot V$ &.$/ 49$ & STS \\
\hline
\end{tabular}

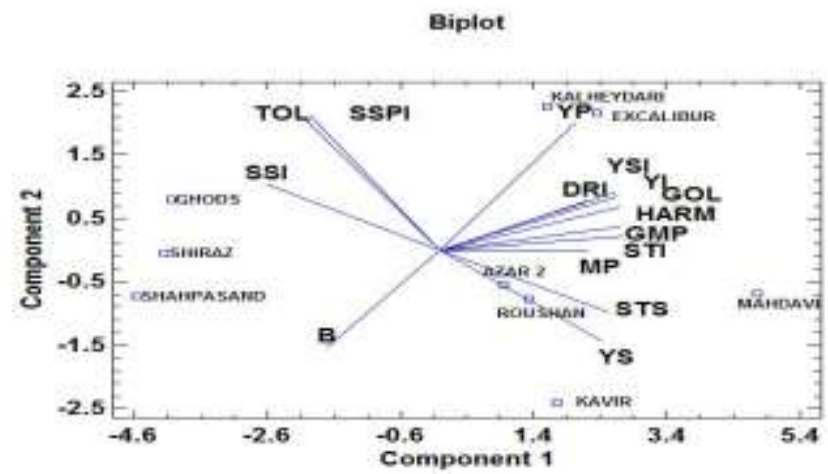

شكل 1- نمايش گرافيكى مولفههاى اصلى اول و دوم براى ارقام گندم و شاخصهاى تحمل به تنش خشكى

Figure 1. The biplot of two principles in bread wheats and drought tolerance indices

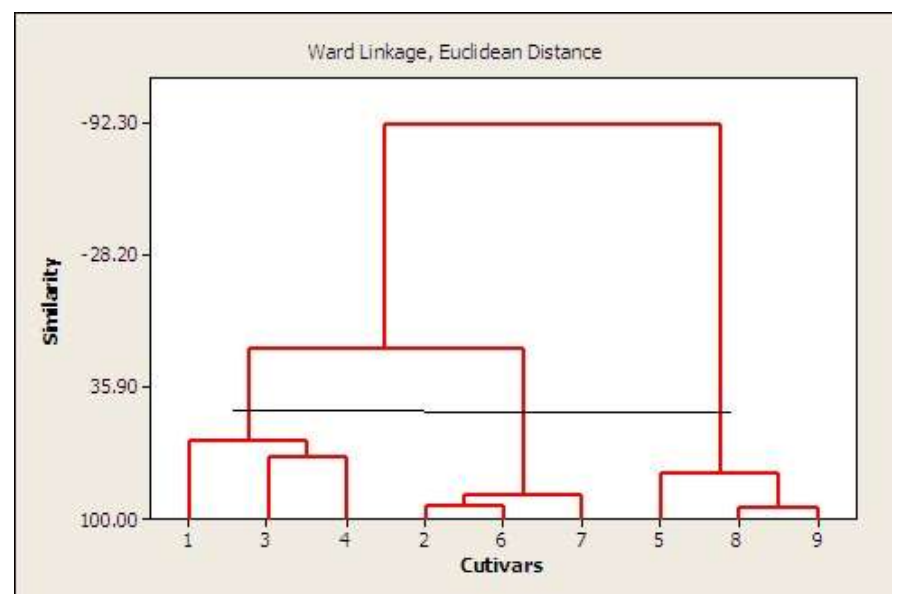

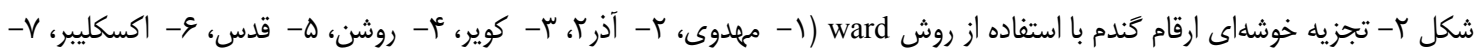
كل حيدرى، ג- شيراز و و

Figure 2.The cluster analysis of bread wheat cultivars according to ward method (1. Mahdavi, 2. Azar2, 3. Kavir, 4. Roushan, 5. Ghods, 6. Excaliber, 7. Kalheydari, 8. Shiraz and 9. Shahpasand). 
1. Abdoshahi, R., A. Safarian, M. Nazari, S.H. Pourseyedi and G.H. Mohammadi-Negad. 2013. Screening drought-tolerant genotypes in bread wheat using different multivariate methods. Archives of Agronomy and Soil Science, 59: 685-704.

2. Ahmadi, A., M. Jodi, A. Tavakoli and M. Ranjbar. 2009. Investigation of yield and its related morphological traits responses in wheat genotypes under drought stress and irrigation conditions. Journal of Crop Production and Processing, 12: 155-165.

3. Akcura, M. and S. Ceri. 2011. Evaluation of drought tolerance indices for selection of Turkish oat (Avena sativa L.) landraces under various environmental conditions. Zemdirbyste Agriculture Journal, 98(2): 157-166.

4. Aminzadeh, G.R. 2010. Evaluation of yield stability of wheat advanced genotypes in Ardabil, Iran. Research Journal of Environmental Sciences, 4(5): 478-482.

5. Anwar, J., G.M. Subhani, M. Hussain, J. Ahmad, M. Hussain and M. Munir. 2011. Drought tolerance indices and their correlation with yield in exotic wheat genotypes. Pakistan Journal of Botany, 43(3): $1527-1530$.

6. Askar, M., A.Yazdansepas and A. Amini. 2010. Evaluation of winter and facultative bread wheat genotypes under irrigated and post-anthesis drought stress conditions. Seed and Plant Improvement Journal, 26(1): 313-329.

7. Boshagh, B., H. Astraki and P. Pezashkipour. 2018. Evaluation of Faba Bean Genotypes using Drought Tolerance Indices and Multivariate Statistical Methods. Journal of Crop Breeding, 10(27): 1-9 (In Persian).

8. Bouslama M., W.T. Jr. Schapaugh. 1984. Stress tolerance in soybean. Part 1: Evaluation of three screening techniques for heat and drought tolerance. Crop Science, 24: 933-937.

9. Dadbakhsh, A. and A. Yazdan-Sepas. 2011. Evaluation of drought tolerance indices for screening bread wheat genotypes in end-season drought stress conditions. Advance Environmental Biology, 5(6): 1040-1045.

10. Fallahi, H.A., J. Alte-Jafarbai and F. Seyedi. 2011. Evaluation of drought tolerance in durum wheat genotypes using drought tolerance indices. Seed and Plant Improvement Journal, 27(1): 15-22.

11. Farshadfar, E., P. Elyasi. 2012. Screening quantitative indicators of drought tolerance in bread wheat (Triticum aestivum L.) landraces. European Journal of Experimental Biology, 2(3): 577-584.

12. Fernandez, G.C.J. 1992. Effective selection criteria for assessing plant stress tolerance. In: Proceeding of the International Symposium on adaptation of vegetable and other food crops in temperature and water stress. Taiwan, pp: 257-270.

13. Finlay, K.W. and G.N. Wilkinson. 1963. The analysis of adaptation in a plant breeding program. Australian Journal of Agricultural Research, 14: 742-754.

14. Fischer, R.A. and R. Maurer. 1978. Drought resistance in spring wheat cultivars. I. Grain responses. Australian Journal of Crop Science, 29: 897-912.

15. Garavandi, M., E. Farshadfar and D. Kahrizi. 2010. Evaluation of drought tolerance in bread wheat advanced genotypes in field and laboratory conditions. Seed and Plant Improvement Journal, 26(1): 233-252.

16. Gholamin, R., M. Zaeifizadeh, M. Khayatnezhad, S. Jamaati-e-Somarin and R. Zabihi-eMahmoodabad .2010. Study of drought tolerance in durum wheat genotypes. American-Eurasian Journal of Agriculture and Environmental Science, 9(5): 465-469.

17. Gholinezhad, E. and R. Darvishzadeh. 2018. Investigation the Drought Tolerance of Sesame (Sesamium indicum L.) Local Landraces Based on Drought Stress Tolerance Indices in Different Levels of Irrigation and Mycorrhizae. Journal of Crop Breeding, 10(26): 185-194 (In Persian).

18. Golbashi, M., M. Ebrahimi, S. Khavari Khorasani and M. H.Saboor. 2011. Investigation of drought tolerance indices in new corn (Zea maize L.) hybrids. Iranian Journal of Field Crops Research, 9(1): 103-113.

19. Ilker, E., F. Tartar Aykut-Tonk and M. Tosun. 2011. Determination of tolerance level of some wheat genotypes to post-anthesis drought. Turkish Journalof Field Crop, 16(1): 59-63.

20. Jafari, A., F. Paknejad and M. Jami-Al-Ahmadi. 2009. Evaluation of selection indices for drought tolerance of corn (Zea mays L.) hybrids. International Journal of Plant Production, 3(4): 33-38.

21. Karimizadeh, R. and M. Mohammadi. 2011. Association of canopy temperature depression with yield of durum wheat genotypes under supplemental irrigation and rainfed conditions. Australian Journal of Crop Science, 5: 138-146.

22. Khakwani, A.A., M.D. Dennett, M. Munir and M. Abid. 2012. Growth and yield response of wheat varieties to water stress at booting and anthesis stages of development. Pakistan Journal of Botany, 44(3): 879-886.

23. Khezri Afravi, M., E.A. Hoseinzadeh, V.A. Mohammadi and E. Ahmadi. 2010. Evaluation of drought resistance in local durum wheat cultivars in Iran under drought stress and natural irrigation conditions. Iranian Journal of Agricultural Sciences, 41(4): 741-753. 
24. Kilic, H. and T. Yagbasanlar. 2010. The effect of drought stress on grain yield, yield components and some quality traits of durum wheat (Triticum turgidum ssp. durum) cultivars. Notulae Botanicae Horti Agrobotanici Cluj-Napoca, 38(1): 164-170.

25. Krishania, S., P. Dwivedi and K. Agarwal. 2013. Strategies of adaptation and injury exhibited by plants under a variety of external conditions: a short review. Comunicata Scientiae, 4(2): 103-110.

26. Kristin, A.S., R.R. Serna, F.I. Perez, B.C. Enriquez, J.A.A. Gallegos and P.R. Vallego. 1997. Improving common bean performance under drought stress. Crop Science, 37: 43-50.

27. Lin, C.S., M. Binns and L.P. Lefkovitch. 1986. Stability analysis: where do we stand? Crop Science, 26: 894-900.

28. Majidi, M.M., V. Tavakoli, A. Mirlohi and M.R. Sabzalian. 2011. Wild safflower species (Carthamus oxyacanthus Bieb): A possible source of drought tolerance for arid environment. Australian Journal of Agricultural Research, 5: 1055-1066.

29. Mohammadi, R., D. Sadeghzadeh, M. Armion and A. Amri. 2011. Evaluation of durum wheat experimental lines under different climate and water regime strategies. Crop and Pasture Science, 62:137-151.

30. Mollasadeghi, V., M. Valizadeh, R. Shahryari and A. Imani. 2011. Evaluation of end drought tolerance of 12 wheat genotypes by stress indices. World Applied Science Journal, 13(3): 545-551.

31. Moosavi, S.S., B. Yazdi Samadi, M.R. Naghavi, A.A. Zali, H. Dashti and A. Pourshahbazi. 2008. Introduction of new indices to identify relative drought tolerance and resistance in wheat genotypes. Desert, 12: 165-178.

32. Nouri, A., A. Etminan, A.D. Jaime, T. Da-Silva and R. Mohammadi. 2011. Assessment of yield, yield-related traits and drought tolerance of durum wheat genotypes (Triticum turgidum var. Durum Desf.). Australian Journal of Crop Science, 5(1): 8-16.

33. Nouri-Ganbalani, A., G. Nouri-Ganbalani and D. Hassanpanah. 2009. Effects of drought stress condition on the yield and yield components of advanced wheat genotypes in Ardabil, Iran. Journal of Food, Agriculture and Environment, 7: 228-234.

34. Pireivatlou, A.S., B.D. Masjedlou and R.T. Aliyev. 2010. Evaluation of yield potential and stress adaptive trait in wheat genotypes under post anthesis drought stress conditions. African Journal of Agricultural Research, 5: 2829-2836.

35. Ranjbar, G.H. and M.J. Rousta. 2010. The most effective stability index for selection of wheat genotypes in saline condition. Soil Research Journal, 24(3): 283-290.

36. Rosielle, A.A., J. Hamblin. 1981. Theoretical aspects of selection for yield in stress and non-stress environments. Crop Science, 21: 943-946.

37. Salehi, M. and S.A. Mosavat. 2008. Selection criteria of wheat genotypes under salt stress in Golestan province. Electronic Journal of Crop Production, 1(4): 19-33.

38. Sio-Se-Mardeha. A., A. Ahmadi, K. Poustini and V. Mohammadi. 2006. Evaluation of drought resistance indices under various environmental conditions. Field Crops Research, 98: 222-229.

39. Sokoto, M.B. and A. Singh. 2013. Yield and yield components of bread wheat as influenced by water stress, sowing date and cultivar in Sokoto, Sudan Savannah, Nigeria. American Journal of Plant Sciences, 4: 122-130.

40. Soleymanifard, A., K.H. Fasihi, H. Nasirirad and R. Naseri. 2010. Evolution of stress tolerance indices in durum wheat genotypes. Journal of Plant Production, 17: 39-58.

41. Spiertz, J.H.J., R.J. Harmer, H. XU, C. Primo-Martinc, C. Don and P.E.1. Vander Putten. 2006. Heat stress in wheat effects on grain weight and quality within genotypes. Europian Journalof Agriculture, 29: 89-95.

42. Talebi, R., F. Fayaz and A.M. Naji. 2009. Effective selection criteria for assessing drought stress tolerance in durum wheat. General and Applied Plant Physiology, 35(1-2): 64-74.

43. Yarnia, M., N. Arabifard, F. Rahmizadeh Khoei and P. Zandi. 2011. Evaluation of drought tolerance indices among some winter rapeseed cultivars. African Journal of Biotechnology, 10: 10914-10922.

44. Zebarjadi, A.R., S. Tavakoli Shadpey, A.R. Etminan and R. Mohammadi. 2013. Evaluation of drought stress tolerance in durum wheat genotype using drought tolerance indices. Seed and Plant Improvement Journal, 29-1: 1-12. 


\title{
Evaluation of Grain Yield, Agronomical Traits and Drought Tolerance Indices in Some Bread Wheat Cultivars
}

\author{
Ali Eftekhari ${ }^{1}$, Amin Baghizadeh ${ }^{2}$, Rooholah Abdoshahi $^{3}$ and \\ Mohammad Mahdi Yaghoubi ${ }^{2}$
}

1- Assistant professor, Department of Agricultural sciences, Payame Noor University, Tehran, Iran

2- Associate Professor, Department of Biotechnology, Institute of Science and High Technology and Environmental Sciences, Graduate University of Advanced Technology, Kerman, Iran

3- Associate Professor, Department of Biotechnology, Institute of Science and High Technology and Environmental Sciences, Graduate University of Advanced Technology, Kerman, Iran Received: June 22, $2018 \quad$ Accepted: May 11, 2019

\begin{abstract}
In order to study genetic variation and effect of drought stress on grain yield, some agronomical traits and 13 drought tolerance indices, an experiment was conducted on nine cultivars at Shahid Bahonar University of Kerman research field. The experimental layout was conducted in a randomized complete block design with three replications under two complementary irrigation and dryland conditions. Results indicated that cultivars and environment treatments significantly affect the yield and the most of the other evaluated traits. Significant reduction was found in grain yield, flag leaf area and seed weight as a result of the drought. According to the results of drought indices and cultivars distribution in biplot, STS, YI, HARM, STI, MP and GMP are the most suitable indices. According to these results, Mahdavi, Kavir and Roushan are tolerant drought cultivars. Therefore, these cultivars can be introduced as a right candidate for the next breeding programs.
\end{abstract}

Keywords: Biplot, Bread Wheat, Drought Stress, Grain Yield, Tolerance Indices 